\title{
PERFIL DE ÁCIDOS GRASOS DE CARNE DE OVINO Y CABALLO CRIADOS BAJO UN SISTEMA DE PRODUCCIÓN EXTENSIVA
}

\author{
Fatty Acid Profile of Sheep and Horse Meat \\ under an Extensive Production System
}

\author{
Lindon W. Mamani-Linares ${ }^{1,2}$, Carmen Gallo ${ }^{1}$
}

\section{Resumen}

Se comparó el contenido de grasa y perfil de ácidos grasos de carne de ovino y caballo finalizados bajo pastoreo. Los ovinos $(n=10)$ y caballos $(n=10)$ fueron faenados bajo procedimientos estándares de Chile. Las canales fueron refrigeradas durante 24 horas a $4{ }^{\circ} \mathrm{C}$. Los análisis fueron realizados en el músculo Longissimus lumborum (LL). La carne de ovino presentó mayor contenido de grasa intramuscular y mayores niveles de ácidos grasos saturados, pero más bajos niveles de ácidos grasos poliinsaturados en comparación a la carne de caballo $(\mathrm{p}<0.05)$. En la relación ácidos grasos poliinsaturados/saturados y el contenido de ácido linoleico conjugado, la carne de ovino presentó valores inferiores a los encontrados para carne de caballo; sin embargo, la carne de ovino mostró la relación n-6/n-3 (2.60) dentro de los niveles recomendados y adecuados niveles de ácidos grasos deseables (67.76\%).

Palabras clave: ácidos grasos, carne, ovino, caballo

\section{Abstract}

This study compared the fat content and fatty acid profile of sheep and horse finished under grazing systems. The sheep $(n=10)$ and horses $(n=10)$ were slaughtered using Chilean standard procedures. Carcasses were stored for $24 \mathrm{~h}$ in a cold room $\left(4^{\circ} \mathrm{C}\right)$. Analyses were carried out on the Longissimus lumborum muscle. Sheep meat had higher content of intramuscular fat and higher levels of saturated fatty acids, but lower levels of polyunsaturated fatty acids compared to horse meat $(\mathrm{p}<0.05)$. In the polyunsaturated to saturated fatty acid ratio and linoleic conjugated

\footnotetext{
${ }^{1}$ Instituto de Ciencia Animal, Facultad Ciencias Veterinarias, Universidad Austral de Chile, Valdivia, Chile

${ }^{2}$ E-mail: willymlmvzupea_2@hotmail.com
}

Recibido: 30 de octubre de 2012

Aceptado para publicación: 7 de abril de 2013 
content, sheep meat presented values lower than those founded for horse meat. However, sheep meat showed an n-6/n-3 (2.60) ratio within the recommended levels and good levels of desirable fatty acids $(67.76 \%)$.

Key words: fatty acids, meat, sheep, horse

\section{INTRODUCCIÓN}

La composición de ácidos grasos y los niveles de colesterol en la carne han recibido una creciente atención debido a sus implicancias en la salud humana y en la calidad del producto. Varios estudios epidemiológicos han asociado el consumo de carne roja y la carne procesada con el desarrollo de dos enfermedades crónicas de importancia en el mundo occidental, las enfermedades cardiovasculares (ECV) y el cáncer de colon (Giovannucci et al., 1994; Kelemen et al., 2005; Cross et al., 2007; Kontogianni et al., 2008). Algunos constituyentes de las carnes rojas han sido propuestos como responsables de estas asociaciones; entre estos, el contenido graso, ácidos grasos y la posible formación de compuestos cancerígenos, como las aminas heterocíclicas (HCA) por cocinado de carne a altas temperaturas (Bingham et al., 2002).

Las proporciones de ácidos grasos poliinsaturados (PUFA) y grasas saturadas (SFA), omega-6 (n-6) y omega-3 (n-3) PUFA, así como hipocolesterolémicos e hipercolesterolémicos $(\mathrm{h} / \mathrm{H})$ son ampliamente utilizadas para evaluar el valor nutricional de las grasas. En las últimas décadas, las investigaciones se han abocado en los efectos de los ácidos grasos individuales sobre el metabolismo de los lípidos y la prevención de las enfermedades coronarias. Recientemente, las investigaciones se han centrado en el ácido linoleico conjugado (CLA) y la proporción de CLA/SFA + colesterol, debido a su actividad anticancerosa. Se dispone de abundante evidencia que apoya el papel de la car- ne roja magra como positivo moderador de los perfiles de lípidos que la identifican como fuente dietética de anti-inflamatorios, cadena larga (LC) n-3 PUFA y el ácido linoleico conjugado (CLA) (McAfee et al., 2010). La composición de ácidos grasos de la carne se encuentra mayormente influenciada por la edad, régimen de alimentación y genotipo del ganado (Huerta-Leindez et al., 1993; De Smet et al., 2004; Alfaia et al., 2006).

La escasez de estudios sobre el valor nutricional de las grasas de carnes no tradicionales como la carne de caballo motivaron el presente estudio, que tuvo como objetivos determinar y comparar el perfil de ácidos grasos de carne de ovino y caballo.

\section{Materiales Y Métodos}

Se trabajó con muestras del músculo Longissimus lumborum (LL), proveniente de 10 ovinos y 10 caballos, entre los meses de mayo y julio de 2010. Las muestras de ovinos fueron obtenidas de corderos machos (cruzas con predominio de razas de carne) de la región de Los Lagos, y que fueron faenados en el matadero de Osorno. Las muestras de caballos proceden de animales faenados de acuerdo a los procedimientos estándares de Chile y adquiridas en carnicerías especializadas, seleccionadas al azar y visitadas en diferentes días, en la ciudad de Temuco, región de La Araucanía, Chile.

Los animales utilizados en el estudio procedieron de zonas con predominio de un sistema de producción extensivo, donde la 
alimentación está basada en praderas nativas o praderas cultivadas naturalizadas. Las canales fueron refrigeradas por 24 horas (0$4{ }^{\circ} \mathrm{C}$ ) previo a la toma de la muestra. Las muestras de las dos especies fueron transportadas en refrigeración al Laboratorio de Carnes del Instituto de Ciencia Animal, Facultad de Ciencias Veterinarias, Universidad Austral de Chile. Cada muestra de carne fue cortada en bifes de $2.5 \mathrm{~cm}$ de espesor, identificadas con códigos individuales y almacenadas a $-20{ }^{\circ} \mathrm{C}$.

El análisis de laboratorio de las muestras se hizo dentro de 60 días de su adquisición. El análisis de composición proximal se hizo en duplicado, utilizando el método oficial 991.36 (AOAC, 1996) para la determinación de contenido de grasa.

Los lípidos de la muestra liofilizada se extrajeron de acuerdo a Folch et al. (1957). El extracto de lípidos fue convertido a ésteres metílicos de ácidos grasos (FAME) usando la metodología descrita por Hartman y Lago (1973). La separación y cuantificación de los ésteres se llevó a cabo utilizando un cromatógrafo de gases (GC-2010 Shimadzu AOC-20i auto inyector, AOC-20s auto sampler Shimadzu) equipado con una columna capilar Rt-2560 (100 m, 0.25 nm ID y 0.20 $\mu \mathrm{m}$ film thickness).

Los ácidos grasos individuales fueron identificados por comparación de sus tiempos de retención con los estándares de la mezcla de ácidos grasos Sulpeco 37 (Sigma Chemical, UK), utilizados como estándares internos. Los ácidos grasos se expresaron como porcentaje del total de ácidos grasos identificados, y fueron agrupados como ácidos grasos saturados (SFA), monoinsaturados (MUFA) y ácidos grasos poliinsaturados (PUFA).

El análisis estadístico de los datos se realizó a través de una comparación de medias, utilizando una prueba $\mathrm{T}$ para muestras independientes. Se utilizó el programa Statistix para Windows v. 8.0.

\section{Resultados y Discusión}

El porcentaje de grasa fue más alto en el ovino $(2.56 \pm 0.41)$ que en el equino $(0.67$ $\pm 0.11)(\mathrm{p}<0.05)$. En general, el contenido de grasa del LD de corderos en el presente estudio fue similar a los valores de 1.76 a $3.97 \%$ reportados para ovinos jóvenes (Perlo et al., 2008), pero diferente a los ovinos adultos (8.79\%) (Göncü Karakök et al., 2010); mientras que en caballos el contenido de grasa fue bajo en relación a otros estudios que describen valores de 2.08 a $4.52 \%$ (Tateo et al., 2008; Lanza et al., 2009).

En el Cuadro 1 se presenta el perfil de ácidos grasos de la carne en las dos especies, observándose diferencias en los niveles de SFA, MUFA y PUFA. En general, el principal ácido graso en la grasa intramuscular fue C18:1n-9, seguido por C16:0. El tercer ácido grado de mayor presencia fue C18:0 en ovinos y C 18:2 n-6 en caballos.

El Cuadro 2 muestra la proporción de ácidos grasos en la grasa intramuscular. Los SFA representan cerca del $45 \%$ del total de ácidos grasos en ovinos, mientras que en caballos fue de $38.9 \%$. Los MUFA fueron ligeramente mayores en ovinos y los PUFA en los caballos.

El nivel de ácido linoleico del músculo Longissimus dorsi en carne de caballo en el presente estudio (10.84\% del total de ácidos grasos) fue mucho más alto comparado con el nivel reportado en bovinos (2.8 a $4.3 \%)$ y en corderos $(4.6 \%)$ criados en diferentes sistemas de producción (Realini et al., 2004; Nürnberg et al., 2005; Lee et al., 2008). Es conocido que el ácido linoleico en los rumiantes es degradado a MUFA y SFA por biohidrogenación microbiana con una pequeña proporción disponible para la deposición en los tejidos lipídicos. En monogástricos, las proporciones de ácido linoleico en el músculo dependen estrictamente de la dieta, debido a que no cambia durante su pasaje a través del estómago, absorción en el intestino delgado e incorporación en los tejidos (Wood et al., 2008). 
Cuadro 1. Contenido de grasa y perfil de ácidos grasos (promedio \pm d.e.) del músculo Longissimus lumborum de ovino y caballo

\begin{tabular}{|c|c|c|c|}
\hline & & Ovino & Caballo \\
\hline \multicolumn{4}{|l|}{ Ácidos grasos saturados } \\
\hline Ac. Butírico & $\mathrm{C} 4: 0$ & $1.70 \pm 0.03^{\mathrm{a}}$ & $0.16 \pm 0.01^{\mathrm{b}}$ \\
\hline Ac. Caproico & C 6:0 & nd & nd \\
\hline Ac. Caprílico & C 8:0 & $0.02 \pm 0.00^{\mathrm{a}}$ & $0.02 \pm 0.01^{\mathrm{a}}$ \\
\hline Ac. Cáprico & C 10:0 & $0.55 \pm 0.02^{\mathrm{a}}$ & $0.07 \pm 0.01^{\mathrm{b}}$ \\
\hline Ac. Láurico & C 12:0 & $0.85 \pm 0.03^{\mathrm{a}}$ & $0.18 \pm 0.02^{b}$ \\
\hline Ac. Tridecanoico & C 13:0 & nd & $0.02 \pm 0.01$ \\
\hline Ac. Mirístico & C 14:0 & $5.71 \pm 0.10^{\mathrm{a}}$ & $3.64 \pm 0.12^{b}$ \\
\hline Ac. Pentadecanoico & C 15:0 & $0.57 \pm 0.01^{\mathrm{a}}$ & $0.15 \pm 0.02^{\mathrm{b}}$ \\
\hline Ac. Palmítico & C 16:0 & $21.29 \pm 3.88^{\mathrm{b}}$ & $30.45 \pm 4.14^{\mathrm{a}}$ \\
\hline Ac. Margárico & C 17:0 & $1.44 \pm 0.11^{\mathrm{a}}$ & $0.30 \pm 0.02^{\mathrm{b}}$ \\
\hline Ac. Esteárico & C 18:0 & $12.53 \pm 1.22^{\mathrm{a}}$ & $4.15 \pm 0.12^{\mathrm{b}}$ \\
\hline Ac. Araquídico & C 20:0 & $0.02 \pm 0.00^{\mathrm{a}}$ & $0.04 \pm 0.01^{\mathrm{a}}$ \\
\hline \multicolumn{4}{|l|}{ Ácidos grasos monoinsaturados } \\
\hline Ac. Miristoleico (sin estándar) & C $14: 1$ & $0.38 \pm 0.03^{\mathrm{a}}$ & $0.20 \pm 0.01^{\mathrm{a}}$ \\
\hline Ac. Miristoleico & C $14: 1 \mathrm{n}-5$ & $0.20 \pm 0.01^{\mathrm{a}}$ & $0.20 \pm 0.02^{\mathrm{a}}$ \\
\hline Ac. Pentad ecaenoico & C $15: 1 \mathrm{n}-5$ & $0.30 \pm 0.03^{\mathrm{a}}$ & $0.06 \pm 0.01^{\mathrm{a}}$ \\
\hline Ac. Palmitelaídico & C $16: 1 \mathrm{t}$ & $2.91 \pm 0.16$ & nd \\
\hline Ac. Palmitoleico & C $16: 1 \mathrm{n}-7$ & $1.52 \pm 0.07^{\mathrm{b}}$ & $9.90 \pm 1.02^{\mathrm{a}}$ \\
\hline Ac. Palmitoleico & C $16: 1$ & nd & $1.04 \pm 0.02$ \\
\hline Ac. Heptadecen oico & C $17: 1$ & $0.66 \pm 0.03$ & $0.20^{\mathrm{a}} \pm 0.02^{\mathrm{a}}$ \\
\hline Ac. trans Vaccénico (trans 11) & C $18: 1 \mathrm{t}$ & nd & nd \\
\hline Ac. Elaídico (trans 9) & C $18: 1$ & $2.82 \pm 0.12$ & nd \\
\hline Ac. Oleico (cis 9) & C $18: 1 \mathrm{n}-9$ & $36.20 \pm 4.11^{\mathrm{a}}$ & $31.98 \pm 4.89^{\mathrm{b}}$ \\
\hline Ac. Vaccénico & C $18: 1 \mathrm{n}-9$ & $0.04 \pm 0.01^{\mathrm{b}}$ & $0.79 \pm 0.03^{\mathrm{b}}$ \\
\hline Ac. Eicose noico & C $20: 1 \mathrm{n}-9$ & $0.04 \pm 0.01^{\mathrm{a}}$ & $0.07 \pm 0.02^{\mathrm{a}}$ \\
\hline \multicolumn{4}{|l|}{ Ácidos grasos poliinsaturados } \\
\hline $\begin{array}{l}\text { Ac. Linolelaídico (6-trans, } 12 \\
\text { trans) }\end{array}$ & C $18: 2 \mathrm{t}$ & nd & nd \\
\hline Ac. Linoleico (cis-9 cis-12) & C $18: 2$ n-6 & $6.92 \pm 1.08^{\mathrm{b}}$ & $10.84 \pm 1.12^{\mathrm{a}}$ \\
\hline Ac. Linoleico (cis-10 cis-12) & C $18: 2$ & nd & nd \\
\hline Ac. Linoleico (cis-9 trans-11) & C $18: 2 n-3$ & $0.89 \pm 0.11$ & nd \\
\hline $\begin{array}{l}\text { Ac. alfa Linolénico (cis-9 cis-12 } \\
\text { cis-15) }\end{array}$ & C $18: 3 n-3$ & $1.07 \pm 0.08^{\mathrm{b}}$ & $5.93 \pm 1.09^{\mathrm{a}}$ \\
\hline Ac. gama Linolénico & C $18: 3$ & nd & nd \\
\hline Ac. Eicosa dienoico & C $20: 2 n-6$ & $0.09 \pm 0.01^{\mathrm{a}}$ & $0.11 \pm 0.03^{\mathrm{a}}$ \\
\hline Ac. Eicosa trienoico & C $20: 3 n-3$ & $0.60 \pm 0.13^{\mathrm{a}}$ & $0.75 \pm 0.12^{\mathrm{a}}$ \\
\hline Ac. Eicosa pentaenoico & C $20: 5 n-3$ & $0.34 \pm 0.04^{\mathrm{a}}$ & $0.14 \pm 0.02^{\mathrm{b}}$ \\
\hline Ac. Docosapentaenoico & C $22: 5 n-3$ & $0.36 \pm 0.03^{\mathrm{a}}$ & $0.24 \pm 0.03^{\mathrm{ab}}$ \\
\hline Ac. Docosahexaenoico & C $22: 6 n-3$ & nd & nd \\
\hline
\end{tabular}

${ }^{a, b}$ Superíndices diferentes dentro de filas indican diferencia estadística $(p<0.05)$. nd: no detectado 
Cuadro 2. Índices de ácidos grasos relacionados a la salud humana del músculo Longissimus lumborum de ovino y caballo

\begin{tabular}{|c|c|c|}
\hline Suma e índices ${ }^{1}$ & Ovino (\%) & Caballo (\%) \\
\hline$\sum S F A$ & 44.8 & 38.9 \\
\hline ¿MUFA & 45.0 & 43.2 \\
\hline SPUAA & 10.3 & 17.9 \\
\hline$\sum \mathrm{CLA}$ & 1.96 & 5.93 \\
\hline$\sum n-3$ & 2.90 & 6.73 \\
\hline$\sum n-6$ & 7.38 & 11.14 \\
\hline$\sum \mathrm{h}$ & 49.20 & 49.90 \\
\hline$\sum H$ & 27.85 & 29.86 \\
\hline$n-6 n-3$ & 2.60 & 1.68 \\
\hline hH & 1.77 & 1.49 \\
\hline DUFASA $A$ & 0.23 & 0.47 \\
\hline$\sum \mathrm{DEA}$ & 67.76 & 65.21 \\
\hline
\end{tabular}

${ }^{1}$ SFA = Ácidos grasos saturados; MUFA = ácidos grasos monoinsaturados; PUFA = ácidos grasos poliinsaturados; CLA = ácido linoleico conjugado; $\mathrm{n}-3=$ omega $3 ; \mathrm{n}-6=$ omega 6 ; $\mathrm{h}=$ hipocolesterolaémicos (suma de 18:1, 18:2, 18:3, 20:5, 22:5, 22:6); H = hipercolesterolaémicos (suma de 12:0, 14:0, 16:0); DFA = ácidos grasos deseables (suma de MUFA+PUFA+C18:0)

En general, animales finalizados sobre forraje tienen más altos niveles de PUFA, ácidos grasos n-3 y CLA que aquellos finalizados con concentrado (Realini et al., 2004; Nürnberg et al., 2005), proveyendo carne con un perfil de ácidos grasos más favorable para el consumidor desde una perspectiva de salud (Scollan et al., 2006). Los lípidos de los forrajes frescos son caracterizados por un predominio de $\mathrm{C} 18: 3$, un precursor de la serie $\mathrm{n}-3$, mientras que los concentrados contienen niveles relativamente más altos de C18:2, precursor de la serie n-6 (Fisher et al., 2000; Velasco et al., 2001).

La grasa intramuscular de caballos tiene una relación más favorable de PUFA:SFA; además, la relación de n-6: n-3 en grasa intramuscular de caballos fue menor que las proporciones de 2 y 3 reportadas para carne de bovinos y ovinos criados en sistemas de pastoreo (USDA, 2008).

La relación de PUFA/SFA, n-6/n-3 PUFA, h/H, 18:0 + 18:1/16:0, CLA/ SFA+colesterol, C20:3n-6 + C20:5n-3/C20:4n6 y la suma de ácidos grasos deseables (DFA) son comúnmente usados para evaluar el valor nutricional y consumo saludable de grasa intramuscular (Orellana et al., 2009). Grasas que presentan bajos PUFA/SFA son consideradas no favorables, porque pueden inducir un incremento de colesterol en la sangre. La grasa de rumiantes, especialmente cuando son criados sobre pastura, presenta valores de PUFA/SFA entre 0.15 a 0.25 (Lee et al., 2008; Dierking et al., 2010). 
La relación PUFA:SFA de grasa intramuscular (IMF) de ovinos fue más baja que el mínimo recomendado (0.4), mientras que la relación n-6:n-3 estuvo dentro de la relación dietaria recomendada (menor de 4) (BDH, 1994).

Una relación n-6/n-3 más baja es deseada para reducir el riesgo de las enfermedades crónicas. Los resultados muestran que del total de ácidos grasos encontrados en la carne de ovinos (Cuadro 2), solo el 32.24\% (100-67.76) es considerado perjudicial para la salud, mientras que en caballos fue el $34.79 \%$. No obstante, estas concentraciones en el músculo Longissimus fueron más bajas que las reportadas para bovinos (36.2\%) y ovinos (38.42\%) (Göncü Karakök et al., 2010).

\section{Conclusiones}

- La carne de caballo presentó niveles más bajos de grasa intramuscular.

- Los caballos presentaron niveles altos de grasas poliinsaturadas, mientras que los ovinos presentaron niveles más bajos de ácidos grasos perjudiciales para la salud.

\section{Literatura Citada}

1. Alfaia CMM, Ribeiro VSS, Lourenço MRA, Quaresma MAG, Martins SIV, Portugal APV. 2006. Fatty acid composition, conjugated linoleic acid isomers and cholesterol in beef from crossbred bullocks intensively produced and from Alentejana purebred bullocks reared according to CarnalentejanaPDO specifications. Meat Sci 72: 425436.

2. AOAC. 1996. Official methods of analysis. $16^{\text {th }}$ ed. Washington DC: Association of Analytical Chemists. $1298 \mathrm{p}$.
3. $[B D H]$ British Department of Health. 1994. Nutritional aspects of cardiovascular disease. Report on health and social subjects, N. ${ }^{\circ}$ 46. London: Her Majesty's Stationery Office. 48 p.

4. Bingham SA, Hughes R, Cross AJ. 2002. Effect of white versus red meat on endogenous $\mathrm{N}$-nitrosation in the human colon and further evidence of a dose response. J Nutr 132: 3522-3525.

5. Cross AJ, Leitzmann MF, Gail MH, Hollenbeck AR, Schatzkin A, Sinha R. 2007. A prospective study of red and processed meat intake in relation to cancer risk. PLoS Medicine 4(12): e345.

6. De Smet S, Raes K, Demeyer D. 2004. Meat fatty acid composition as affected by fatness and genetic factors: A review. Animal Res 53: 81-98.

7. Dierking R, Kallenbach R, Grün I. 2010. Effect of forage species on fatty acid content and performance of pasture finished steers. Meat Sci 85: 597-605.

8. Fisher AV, Enser M, Richardson RI, Wood JD, Nute GR, Kurt E. 2000. Fatty acid composition and eating quality of lamb types derived from four diverse breed production systems. Meat Sci 55: 141-147.

9. Folch J, Lees M, Stanley S. 1957. A simple method for the isolation and purification of total lipids from animal tissues. J Biol Chem 226: 497-509.

10. Giovannucci E, Rimm EB, Stampfer MJ, Colditz GA, Ascherio A, Willet WC. 1994. Intake of fat, meat and fiber in relation to risk of colon cancer in men. Cancer Res 54: 2390-2397.

11. Göncü Karakök S, Ozogul Y, Saler M, Ozogul F. 2010. Proximate analysis. Fatty acid profiles and mineral contents of meats: a comparative study. J Muscle Foods 21: 210-223.

12. Hartman L, Lago R. 1973. Rapid preparation of fatty acid methyl esters from lipids. Lab Pract 22: 475-476.

13. Huerta-Leindez, NO, Cross HR, Savell JW, Lunt DK, Baker JF, Pelton LS. 1993. Comparison of the fatty acid composition of subcutaneous adipose 
tissue from mature Brahman and Hereford cow. J Anim Sci 71: 625-630.

14. Kelemen LE, Kushi LH, Jacobs DR, Cerhan JR. 2005. Associations of dietary protein with disease and mortality in a prospective study of postmenopausal women. Am J Epidemiol 161: 239-249.

15. Kontogianni MD, Panagiotakos DB, Pitsavos C, Chrysohoou C, Stefanadis C. 2008. Relationship between meat intake and the development of acute coronary syndromes: The CARDIO2000 case-control study. Eur J Clin Nutr 62: 171-177.

16. Lanza M, Landi C, Scerra M, Galofaro V, Pennini P. 2009. Meat quality and intramuscular fatty acid composition of Sanfratellano and Haflinger foals. Meat Sci 81: 142-147.

17. Lee J, Kannan G, Eega K, Kouakou B, Getz W. 2008. Nutritional and quality characteristics of meat from goats and lambs finished under identical dietary regime. Small Ruminant Res 74: 255259.

18. McAfee A, McSorley E, Cuskelly G, Moss B, Wallace J, Bonham M, Fearon A. 2010. Red meat consumption: An overview of the risks and benefits. Meat Sci 84: 1-13.

19. Nürnberg $K$, Dannerberger $D$, Nürnberg G, Ender K, Voigt J, Scollan ND. 2005. Effect of grass-based and concentrate feeding system on meat quality characteristics and fatty acid composition of longissimus muscle in different cattle breeds. Livest Prod Sci 94: 137-147.

20. Orellana C, Peña F, García A, Perez $J$, Martos J, Domenech V, Acero R. 2009. Carcass characteristics, fatty acid composition, and meat quality of Criollo Argentino and Braford steers raised on forage in a semi-tropical region of Argentina. Meat Sci 81: 57-64.

21. Perlo F, Bonato P, Teira G Tisocco O, Vicentin J, Pueyo J, Mansilla A. 2008. Meat quality of lambs produced in the Mesopotamia region of Argentina finished on different diets. Meat Sci 79: 576-581.

22. Realini CE, Duckett SK, Brito GW, Dalla Rizza M, De Mattos D. 2004. Effect of pasture vs. concentrate feeding with or without antioxidants on carcass characteristics, fatty acid composition, and quality of Uruguayan beef. Meat Sci 66: 567-577.

23. Scollan N, Hocquette JF, Nuernberg $K$, Dannenberger D, Richardson I, Moloney A. 2006. Innovations in beef production systems that enhance the nutritional and health value of beef lipids and their relationship with meat quality. Meat Sci 74: 17-33.

24. Tateo A, De Palo $P$, Ceci $E$, Centoducati P. 2008. Physicochemical properties of meat of Italian Heavy Draft horses slaughtered at the age of 11 months. J Anim Sci 86: 1205-1214.

25. [USDA] United States Department of Agriculture. 2008. National nutrient database for standard reference, Release 21. [Internet]. Available in: http:/ /www.ars.usda.gov/ba/bhnrc/ndl

26. Velasco $S$, Cañeque V, Pérez $C$, Lauzurica S, Díaz MT, Huidobro F, Manzanares C, González J. 2001. Fatty acid composition of adipose depots of suckling lambs raised under different production systems. Meat Sci 59: 325-333.

27. Wood JD, Enser M, Fisher AV, Nute GR, Sheard PR, Richardson RI, Hughes SI, Whittington FM. 2008. Fat deposition, fatty acid composition and meat quality: A review. Meat Sci 78: 343-358. 